\title{
Dialoguer pour mieux avancer
}

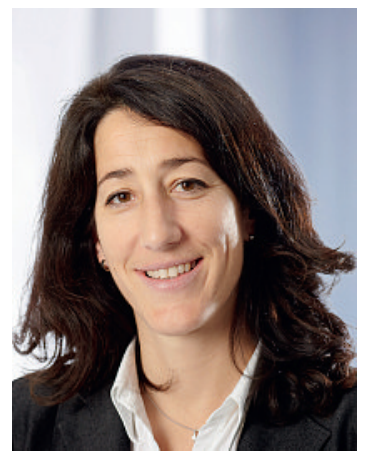

Chères lectrices,

Chers lecteurs,

Lorsque j'ai rejoint le domaine de la santé, après plusieurs expériences au sein de l'administration publique, de l'économie privée, mais également du monde des associations, un défi de taille mais alléchant s'est offert: un domaine à découvrir, une politique publique à élaborer, un service public à restructurer et à repositionner, une grande équipe à piloter, des activités à organiser et diriger, ou encore un système de qualité à mettre en place. Rapidement, les défis du système de santé sont devenus mon quotidien et les acteurs du réseau de santé mes partenaires. Parmi ces derniers, les médecins.

Une telle expérience professionnelle permet de développer une identification forte aux enjeux de politique nationale de la santé et un intérêt accru à participer à son développement et à son avancement. Même si du point de vue de l'autorité le débat sur la maîtrise économique des coûts est prédominant, les questions liées au vieillissement de la population et à ses conséquences sur le système de santé et son organisation, celles liées notamment à la formation des professionnels et la pénurie à venir, à la qualité des prestations, et à la

\section{Seul le dialogue entre tous les acteurs de la santé peut contribuer à l'identification de solutions acceptables pour tous.}

réorientation des pratiques, ne m'ont pas moins occupées. Il en va de même pour ce qui touche à la prévention et à la promotion de la santé, à la recherche et l'innovation ainsi qu'aux nouvelles technologies informatiques.

Et force a été de constater les difficultés récurrentes à trouver un terrain de discussion entre autorités politiques, administrations publiques et médecins. Si j'ai choisi de me tourner vers le monde des médecins et de me mettre au service de la FMH, c'est que ces derniers sont des partenaires privilégiés de toute réforme de la politique sanitaire. Le médecin est un acteur incontournable de la maîtrise des dépenses, car quel que soit les outils qu'on introduit, réseau de soins, mécanisme de coordination, carte de santé, il doit adhérer aux solutions et les porter. Il doit prendre part aux discussions par exemple sur les questions des progrès technologiques, de maîtrise des coûts ou des primes d'assurancemaladie. Seul ce dialogue entre tous les acteurs de la santé peut contribuer à l'identification de solutions acceptables pour tous, servir les intérêts de la population, et par là-même l'image des médecins.

Afin de contribuer à ce dialogue, ma nouvelle fonction au Secrétariat général me donnera sans doute la possibilité

\section{Il m'apparaît indispensable d'aller au-devant des sociétés cantonales et des sociétés de discipline.}

de modifier des approches, revisiter une équipe et son fonctionnement, introduire une nouvelle politique de management et de services, le tout dans un état d'esprit visant à la convergence vers des positions communes. Ma tâche reviendra, entre autre, à analyser la structure actuelle, identifier les principaux besoins, détecter les éventuelles difficultés, rechercher des solutions adaptées et mettre en place des outils de suivi efficaces. Une bonne conduite opérationnelle et une bonne coordination des services centraux doit permettre au Secrétariat général de mener des projets transversaux liés au déploiement stratégique de l'activité de la FMH. Privilégiant le contact et l'échange, il m'apparaît par ailleurs indispensable d'aller au-devant notamment des sociétés cantonales et des sociétés de discipline et de développer une activité et une collaboration étroite avec elles, en tenant compte des besoins de toute la profession. J'entends donc mettre particulièrement l'accent sur cette collaboration ainsi que sur le service que la société faîtière offre à ses membres. Enfin, dans le but de parvenir à une réelle transformation de l'échange entre le niveau stratégique de la FMH et sa partie opérationnelle, il s'agira d'établir une bonne coordination avec l'organe dirigeant de la FMH, et de proposer des outils d'analyses et de conseil de qualité.

Aujourd'hui, je me réjouis donc beaucoup de relever ce défi et de rejoindre l'équipe du Secrétariat général.

Anne-Geneviève Bütikofer, lic. iur. Secrétaire générale de la FMH 